\title{
Curva ambiental de Kuznets: implicaciones para un crecimiento sustentable
}

\author{
Environmental Kuznets Curve: Implications for Sustainable Growth
}

Horacio Catalán*

\section{Resumen}

Este artículo tiene por objetivo realizar una estimación de la curva ambiental de Kutnez para un conjunto de 144 países en el periodo de 1990-2010, mediante la especificación de un modelo de datos panel. Los principales resultados muestran que, la relación entre las emisiones per cápita de $\mathrm{CO}_{2}$ y el PIB per cápita describen una curva en forma de $\mathrm{N}$, indicando que los beneficios que se puedan lograr, en cuanto a la disminución de emisiones de $\mathrm{CO}_{2}$, debido a un mayor crecimiento económico son transitorias. Variables como la eficiencia energética y la protección de los recursos naturales, como parte de la política ambiental, juegan un papel relevante en reducir los impactos del crecimiento económico en el deterioro ambiental.

Palabras clave:

- Emisiones $\mathrm{CO}_{2}$

- Crecimiento económico

- Datos de panel

\section{Abstract}

The objective this article is examine the empirical relation between $\mathrm{CO}_{2}$ emissions per capita and GDP per capita during the period 1990-2010, using a panel of 144 countries. Under the hypothesis the Environmental Kutnez Curve (EKC). The main results show that the relationship between per capita $\mathrm{CO}_{2}$ emissions and per capita GDP describe an Nshaped curve, which means that any beneficial effects economic growth may have on per-capita pollution is transitory. Variables such as energy efficiency and protection of natural resources, play an important role in reducing the impact of economic growth on environmental degradation.

Keywords:

- $\mathrm{CO}_{2}$ Emissions

- Growth

- Panel Data

JEL: C13, Q30, O13

\section{Introducción}

La comprobación empírica de la relación entre crecimiento económico y el deterioro del medio ambiente, ha sido un tema de intenso debate, y la mayoría de las investigaciones se han realizado en el marco de la hipótesis de la curva ambiental de Kuznets (EKC por las siglas en inglés). La cual establece que la relación entre el nivel de ingreso per cápita y el deterioro de la calidad del medio ambiente se representa por una curva con forma de U invertida (Grossman y Krueger, 1993 y 1995; Stern, 1998), indicando que bajos niveles de ingreso están correlacionados con un creciente deterioro en el medio ambiente, pero después de un cierto punto de inflexión del ingreso per cápita, la relación entre las dos variables se vuelve negativa. Así, un mayor nivel de ingreso per cápita corresponde a una disminución en la degradación ambiental (Grossman y Krueger, 1995). En este sentido, la EKC se acota a una comprobación empírica entre indicadores de degradación ambiental e ingreso per cápita.

\footnotetext{
* Profesor de la Facultad de Economía de la unam, Ciudad Universitaria, México, D. F. C.P. 04510 Cub. 20, Email: catalanh@unam.mx catalanh@economia.unam.mx
} 


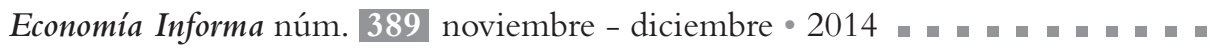

Esta hipótesis se apoya, en el argumento de que mayores niveles de desarrollo implican un cambio en la estructura de la economía en favor de la industria y servicios, donde los procesos de producción, se basan en tecnologías más eficientes, que ayudan a conservar los recursos naturales, y de esta manera reducir de manera importante el deterioro del medio ambiente (Grossman y Krueger, 1995). De igual forma, la EKC muestra el desarrollo de una economía a través del tiempo. En una primera fase, es una economía basada en el sector agrícola con un fuerte impacto en la calidad del medio ambiente; en una segunda fase se desarrolla la industria, que si bien se genera un mayor nivel de riqueza tiene como consecuencia un mayor deterioro en la calidad del medio ambiente. Después de un punto de inflexión, la economía sustenta su crecimiento en tecnologías eficientes y más limpias, principalmente en el sector servicios.

Considerando un conjunto de países la hipótesis de la EKC, establece que los países en desarrollo se ubican en la pendiente positiva de la curva, donde sus acciones y políticas en favor del crecimiento generan un deterioro en el medio ambiente, pero al llegar a un estado mayor de desarrollo, implementan políticas para mejorar la calidad del medio ambiente y proteger los recursos naturales, y eventualmente la degradación comienza a disminuir (Nahman y Antrobus, 2005, Andreoni y Levinson, 2001). Este resultado, podría sugerir que el crecimiento económico es la clave para salir de los problemas ambientales. Toda vez, que cuando la economía rebaza ese punto de inflexión los indicadores de deterioro ambiental tendrían que revertirse. No obstante, la evidencia empírica internacional disponible sobre este debate es ciertamente compleja e incluso contradictoria (Nahman y Antrobus, 2005; Grossman y Krueger, 1995; Kaufmann, et al., 1998; Selden y Song, 1994; Bruyn, et al., 1998; Stern y Common, 2001; Galeotti, et al., 2006; Lessmann, 2014).

Los resultados dependen del indicador que mide el deterioro ambiental, el periodo considerado, si las observaciones son de sección cruzada o de panel, así como el nivel de ingreso per cápita considerado (Ekins, 1997; Stern 2003; Dianda, 2004). No obstante ello, puede argumentarse que existen patrones regulares entre el producto per cápita y la degradación ambiental, pero esto no es automático o determinístico, debido a que adquiere diversas formas y depende de factores tales como: la innovación tecnológica, las instituciones y las regulaciones en materia ambiental. El objetivo del presente artículo es comprobar empíricamente la existencia de una curva ambiental Kuznets (EKC), para un conjunto de 144 países utilizando técnicas de estimación de datos panel. El trabajo se integra por cuatro apartados, el primero corresponde a 
la presente introducción. En la segunda sección, se expone la hipótesis teórica que sustenta la EKC, en la tercera parte se presenta los resultados de la evidencia empírica y finalmente las conclusiones.

\section{Marco general de la Curva de Kuznets}

La relación entre el crecimiento económico y el medio ambiente es uno de los temas más intensamente debatidos en la economía ambiental (Beckerman, 1992; Grossman y Krueger, 1995; Kaufmann et al., 1998; Selden y Song, 1994; Bruyn, et al., 1998; Stern y Common, 2001; Nahman y Antrobus, 2005; Galeotti, et al., 2006; Lessmann, 2014). La curva ambiental de Kuznets $^{1}$ (EKC) se ha convertido en una de las principales hipótesis para explicar esta relación (Nahman y Antrobus, 2005). La EKC propone que existe una relación, en forma de $\mathrm{U}$ invertida, entre la degradación del medio ambiente y el ingreso per cápita como se muestra en la Figura 1 (Stern y Common, 1996; Dianda, 2005).

\section{Figura I}

\section{Curva ambiental de Kuznets}

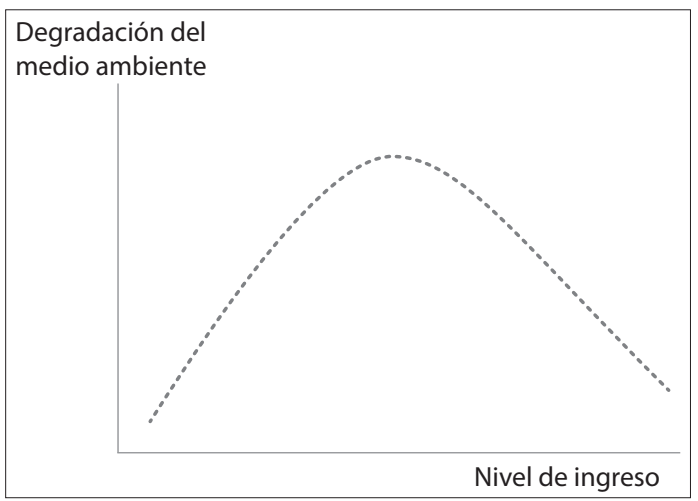

La EKC establece una relación dinámica entre el ingreso per cápita y la calidad del medio ambiente. La calidad del medio ambiente sufre un deterioro en las fases iniciales del proceso de crecimiento, debido a que se intensifica la agricultura y la explotación de los recursos naturales, las tecnologías eficientes y

\footnotetext{
${ }^{1}$ Esta curva debe su nombre al trabajo de Kuznets (1955) que postula una relación en forma de Uinvertida entre el ingreso per cápita y la desigualdad en la distribución del ingreso. Desde principios de los noventa inicia un renovado interés por aplicar esta hipótesis a la relación entre crecimiento económico y degradación ambiental, basada en el estudio pionero de Grossman y Krueger (1991).
} 


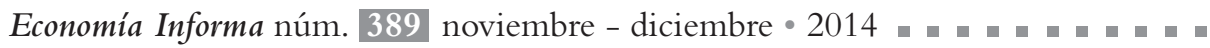

limpias no están disponibles. La extracción de los recursos naturales excede a su conservación y la cantidad de residuos aumenta, este comportamiento se presenta en los países de bajo nivel de desarrollo. La relación entre deterioro ambiental e ingreso per cápita llega a un punto de inflexión donde el deterioro ambiental se detiene y los ingresos siguen aumentando (Rothman, 1998). Este cambio en la relación se explica por un efecto de escala, es decir por el aumento en el nivel de ingreso de la población por un factor " $\mathrm{k}$ " (Panayotou, 1993), que aumenta la disponibilidad de bienes manteniendo contante la degradación ambiental.

Sin embargo, el impacto más importante es por un cambio en la composición del producto, en favor de sectores de la industria y servicios (Stern, 2003). Al interior de la economía las ramas de la actividad económica tienen diferentes intensidades de emisiones de contaminantes, y al modificarse la estructura en favor de estos sectores con menores intensidades de emisiones, se promueve un cambio hacia tecnologías más limpias. Estas modificaciones favorecen una mayor eficiencia en la producción, en consecuencia el deterioro ambiental se detiene y después comienza a revertirse. De este modo, se argumenta (Selden y Song, 1994) que la U invertida es consecuencia de: las elasticidades positivas ${ }^{2}$ entre ingreso y calidad ambiental, de los cambios en la composición del producto favorables al medio ambiente, de las nuevas tecnologías, las presiones asociadas a la mayor información y educación sobre las consecuencias ambientales, y de políticas públicas orientadas a la conservación de los recursos naturales (Selden y Song, 1994; McConnell, 1997; Rothman y Bruyn, 1998).

En este sentido, desde el ámbito de la teoría económica, la calidad ambiental se puede representar como un bien normal por lo que su demanda aumenta al elevarse el ingreso per cápita, lo que se manifiesta también en un cambio en la estructura de preferencias de la demanda en favor de los bienes que generan un menor impacto en el medio ambiente (más amigables ambientalmente) y por una presión creciente por regulaciones ambientales más estrictas (Dianda, 2005). La curva de Kuznets representa entonces, una forma reducida ${ }^{3}$ que encubre otros fenómenos como la tecnología, la composición del producto, las

\footnotetext{
${ }^{2}$ La evidencia internacional sobre la intensidad de insumos ambientales sostiene que existe también una $\mathrm{U}$ invertida conocida como la hipótesis de intensidad de uso o que al menos existe actualmente una tendencia a reducirse esta intensidad asociado a las mejoras tecnológicas que hacen a la producción más eficiente o genera substitutos y a cambios en la estructura productiva (Tilton 1990 y Bruyn, 1997 y Goldemberg, 1992).

${ }^{3}$ Así, las variables incluidas son sólo proxies por lo que puede existir un problema de variables omitidas (Torras y Boyce, 1998)
} 
regulaciones ambientales o las demandas de la sociedad (Grossman y Krueger, 1995; Bruyn, 1997). En este sentido, esta forma reducida no permite identificar inicialmente los efectos de la política económica. No obstante, es posible definir una forma paramétrica de la hipótesis de la EKC (Ekins, 1997; Grossman y Krueger, 1995; Selden y Song, 1994; Suri y Chapman, 1998) definida como:

(1) $E_{i t}=\beta_{0}+\beta_{1} Y P_{i t}+\beta_{2} Y P_{i t}^{2}+\beta_{3} Y P_{i t}^{3}+\sum_{j=1}^{k} \gamma_{j} X_{j, i t}+u_{i t}$ $i=1,2, \ldots N \quad t=1,2, \ldots T$

Donde $E_{i t}$ representa el deterioro ambiental, $Y P_{i t}$ es el producto per cápita, $X_{j, i t}$ es un conjunto de variables que inciden en el deterioro ambiental, tales como: densidad de población (Kaufmann, et al, 1998), grado de apertura comercial, la estructura productiva ${ }^{4}$ (Moonmaw y Unruh, 1997; Panayotou, 1997), emisiones por kilómetro cuadrado (Bruyn, 1997) o incluso variables rezagadas $^{5}$ (Panayotou, 1997; Grossman y Krueger, 1995). Estas variables tienen por objeto capturar el efecto escala, efecto composición y efecto de la tecnología (Grossman, 1995; He y Wang, 2012). El análisis de la descomposición de la EKC en este tipo de efecto se desarrollado por Grossman y Kruger (1995). El deterioro ambiental es un subproducto de la actividad económica, en consecuencia se puede descomponer en un efecto escala, que se refiere al crecimiento de la economía; un efecto composición que es un cambio en la estructura de la economía y un efecto tecnología, que aumentan la eficiencia en el uso de la energía y generan menores niveles de contaminantes (Stern, 2003). La variable $u_{i t}$ representa el término de error. Los subíndices it indican observaciones para distintos individuos (países) y periodos de tiempo, es decir observaciones de datos de Panel.

La ecuación (1) es una forma funcional que permite definir a las siguientes tipos de relaciones entre calidad ambiental e ingreso per cápita (Ekins, 1997; Selden y Song, 1994; Bruyn, Bergh y Opschoor, 1998):

1. Si la eKC cumple con la restricción $\beta_{1}>0$ y $\beta_{2}=\beta_{3}=0$, entonces la relación es lineal y directa de modo que el crecimiento económico se traduce en un mayor deterioro del medio ambiente (es monótona creciente).

2. Se cumple la restricción $\beta_{1}<0$ y $\beta_{2}=\beta_{3}=0$, implica que la relación es lineal e inversa de modo que el crecimiento se traduce en una disminución del deterioro ambiental (es monótona decreciente).

\footnotetext{
${ }^{4}$ Medido por ejemplo como la razón de industria a producto y teniendo mayor peso en los países en vías de desarrollo (Bruyn, 1997).

${ }^{5}$ Las estimaciones de Panayotou (1997) indican que las variables rezagadas no son estadísticamente significativas.
} 


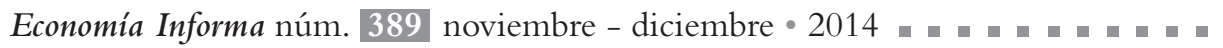

3. Una tercera restricción $\beta_{1}>0, \beta_{2}<0$ y $\beta_{3}=0$, define la relación de $\mathrm{U}$ invertida y existe un punto de inflexión que puede determinarse como $\left(-\beta_{1} / 2 \beta_{2}\right)$. 4. Con $\beta_{1}>0, \beta_{2}<0$ y $\beta_{3}>0$ entonces la forma de la relación es una $N$ abierta. Implicando que un mayor nivel de ingreso per cápita no genera una reducción en el deterioro ambiental.

La Figura 2, representa las distintas formas que puede adoptar la relación ingreso per cápita y deterioro ambiental, considerando las restricciones en los coeficientes de la ecuación (1). Las dos primeras figuras muestran un comportamiento monótono creciente y decreciente, respectivamente (Figura $2 \mathrm{~A}$ y B). Sin embargo, la EKC puede aproximarse mejor a través de un patrón no lineal ya sea a través de una curva en forma de U-invertida o en una curva en forma de N, como se aprecia en la Figura 2 (C y D). Estos patrones más complejos son posibles (especialmente cuando se tienen en horizontes temporales más largos), además la curva-N que puede ser considerado como combinaciones de las cuatro curvas básicas (Bruyn, Bergh y Opschoor, 1998)

\section{Figura 2}

Restricciones en la estimación de la EKC

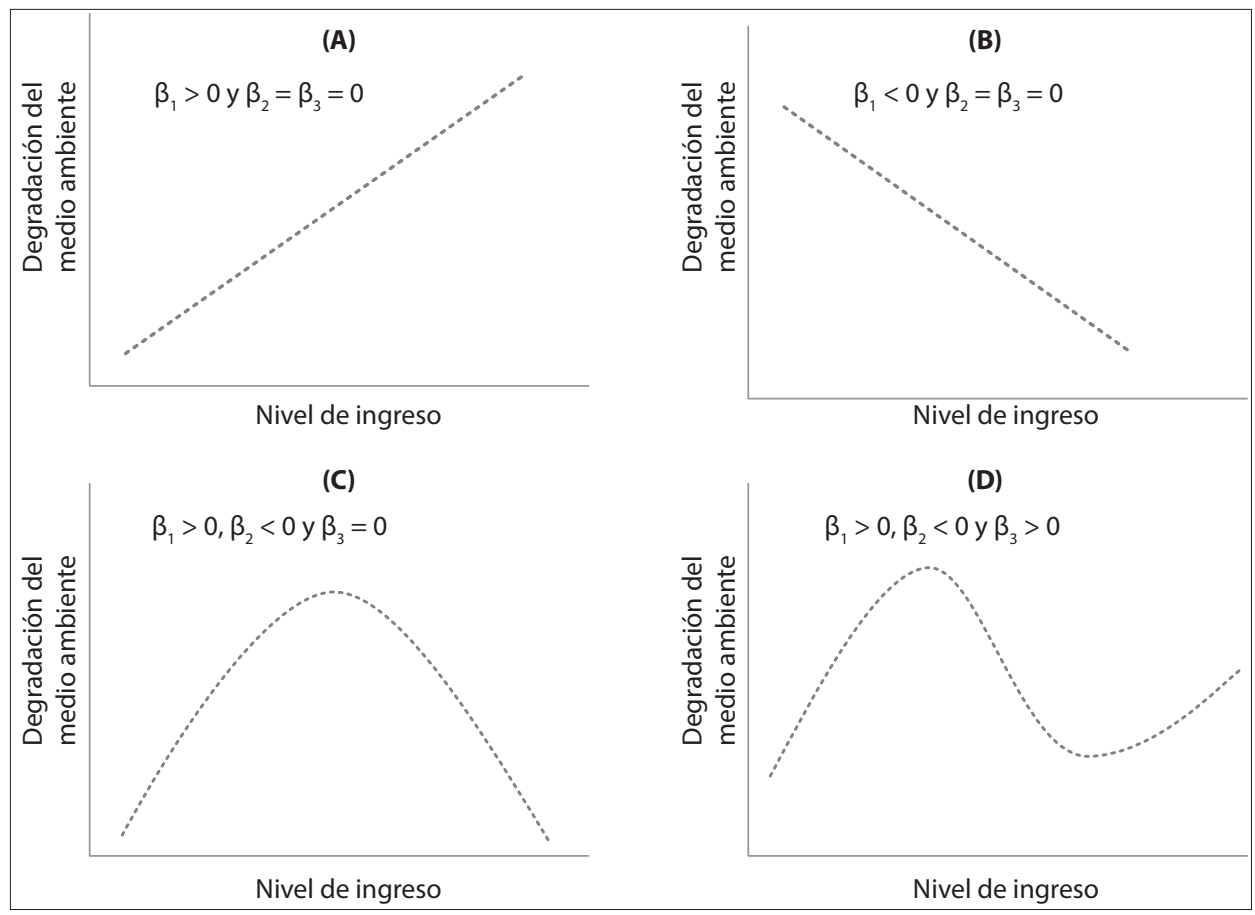


La estimación de la ecuación (1) se puede realizar por mínimos cuadrados ordinarios (POLS). Sin embargo, al considerar un modelo con datos panel que combina información en el tiempo y en sección cruzada (países), existe heterogeneidad en las observaciones de sección cruzada que no puede ser medida o efectos individuales no observables (Greene, 2000; Hsiao, 2003). Esto genera que los estimadores de Pols sean sesgados e inconsistentes. La forma de incluir los efectos no observables es definiendo un modelo de componente de error, donde el error comprende la suma de dos componentes: un término aleatorio y un segundo componente que representa la heterogeneidad no observada. El concepto de heterogeneidad, se puede entender como características propias en las unidades de sección cruzada (países) que no pueden ser medidas y en consecuencia no se pueden estimar. La especificación convencional de los modelos de datos panel con heterogeneidad se representa como:

(2) $y_{i t}=x_{i t}^{\prime} \beta+c_{i}+\varepsilon_{i t} \quad i=1, \ldots, N \quad t=1, \ldots, T$

en donde $x_{i t}$ es un vector de $k$ variables explicativas, $\beta$ es el vector de parámetros del modelo, $c_{i}$ es la heterogeneidad no observable en las unidades de sección cruzada y $\varepsilon_{i t}$ es el término aleatorio que tiene media cero y varianza constante (Greene, 2000). Se asume que la heterogeneidad no observada permanece constante en el tiempo y solo cambia a través de las unidades de sección cruzada (Hsiao, 2003; Baltagi, 2005). La teoría econométrica (Hsiao, 2003; Baltagi, 2005; Greene, 2000) estable dos especificaciones básicas para el modelo de datos panel con heterogeneidad no observable, denominados como efectos fijos y efectos aleatorios.
$y_{i t}=x_{i t}^{\prime} \beta+\alpha_{i}+\varepsilon_{i t}$
Efectos Fijos
(4) $y_{i t}=x_{i t}^{\prime} \beta+v_{i}+\varepsilon_{i t}$
Efectos Aleatorios

La especificación de efectos fijos asume que la heterogeneidad no observable está correlacionado con las variables explicativas $E\left[x_{i t}{ }^{\prime} \alpha_{i}\right] \neq 0$, en consecuencia la estimación de la ecuación (3) puede realizarse por medio de incluir una contante para cada país o individuo. ${ }^{6}$ En el caso de efectos aleatorios se asume que la heterogeneidad no está correlacionada con las variables explicativas

\footnotetext{
${ }^{6}$ Este procedimiento se conoce como mínimos cuadrados ordinarios con variables dummy (LSDV). También se puede realizar la estimación eliminando el término $\alpha_{i}$ ya sea estimado el modelo en primeras diferencias o bien transformando las variables calculado sus desviaciones respecto a su promedio en el tiempo.
} 
Economía Informa núm. 389 noviembre - diciembre • 2014 - "

$E\left[x_{i t}{ }^{\prime} v_{i}\right]=0$, pero sigue una distribución de probabilidad ${ }^{7}$ con media cero $\mathrm{y}$ varianza constante $v_{i} \sim$ iid $\left(0, \sigma_{v}{ }^{2}\right)$ la estimación de la ecuación (4) depende de la covarianza de los términos $v_{i}$ y $\varepsilon_{i t}$. Debido a que $\sigma_{v}^{2}$ y $\sigma_{\varepsilon}^{2}$ no son observados directamente, es necesario obtener primero una estimación de la varianza de cada término, por lo cual se utiliza una estimación de mínimos cuadrados generalizados factibles (FGLS).

Formas alternativas de estimar una ecuación con datos en panel, considerando la presencia de efectos fijos no observables en los datos, es la especificación de media de grupos o between (Greene, 2000), calculando el promedio de las observaciones en series de tiempo en la ecuación (4):

(5) $\bar{y}_{i}=\bar{x}_{i}^{\prime} \beta+\alpha_{i}+\bar{\varepsilon}_{i}$

Media de Grupos (Between)

En la ecuación (5) el término de error se puede definir como $w_{i}=\alpha_{i}+\bar{\varepsilon}_{i}$, es decir ahora la ecuación se denota $\bar{y}_{i}=\bar{x}_{i}{ }^{\prime} \beta+w_{i}$. El estimador de media de grupos (between) es insesgado pero no eficiente, sin embargo al agrupar los datos permite recoger los efectos no observados. Finalmente, la estimación en primera diferencia elimina la heterogeneidad no observada, en efecto al rezagar un periodo la ecuación (2) y restando este resultado a la misma ecuación (2) se obtiene:

(6) $y_{i t}-y_{i t-1}=x_{i t}^{\prime} \beta-x_{i t-1}^{\prime} \beta+\alpha_{i}-\alpha_{i}+\varepsilon_{i t}-\varepsilon_{i t-1}$

(7) $\Delta y_{i t}=\Delta x_{i t}^{\prime} \beta+\Delta \varepsilon_{i t} \quad$ Primera diferencia (FD)

La estimación de la ecuación (7) en primeras diferencias se puede realizar por mínimos cuadrados ordinarios y tiene propiedades adecuadas en los estimadores. No obstante se pierden observaciones de series de tiempo y la interpretación de los resultados debe modificarse al considerarse la variación en las variables del modelo.

\footnotetext{
${ }^{7}$ Asumiendo que los efectos individual no observados $v_{i}$ y el término de error $\varepsilon_{i t}$ se distribuyen como una normal $v_{i} \sim N\left(0, \sigma_{v}{ }^{2}\right)$ y $\varepsilon_{i} \sim N\left(0, \sigma_{\varepsilon}^{2}\right)$, se puede realizar una estimación por máxima verosimilitud (Baltagi, 2005; Greene, 2000). Los resultados entre efectos aleatorios y máxima verosimilitud son bastante similares.
} 


\section{Evidencia empírica}

La información utilizada en el presente artículo son observaciones de datos panel para el periodo de 1990 a 2010, considerando un conjunto de 144 países, la fuente de información es el Banco Mundial, ${ }^{8}$ como la variable de deterioro ambiental se considera el nivel de emisiones de dióxido de carbono $\left(\mathrm{CO}_{2}\right)$ per cápita $\left(\mathrm{CO}_{2 i t}\right)$, medido en toneladas métricas por persona. La variable de ingreso es aproximado por el PIB per cápita medido en dólares de Estados Unidos a precios de $2005\left(y p_{i t}\right)$. Las variables adicionales en la estimación de la EKC corresponden: la participación porcentual del sector servicios en el PIB $\left(\operatorname{serv}_{i t}\right)$; el porcentaje de consumo de energía de origen fósil respecto al total de consumo de energía $\left(\mathrm{ffe}_{i t}\right)$ y la variable de eficiencia energética $\left(\mathrm{efe}_{i t}\right)$, la cual se define como el reciproco de la intensidad energética; 9 y el porcentaje de áreas protegidas respecto al total del territorio $\left(\operatorname{aprot}_{i t}\right)$.

El Cuadro 1, presenta un resumen de los principales estadísticos de las variables utilizadas en la estimación de la EKC, se aprecia que existe una gran dispersión entre las unidades de sección cruzada (países), principalmente en los niveles de ingreso per cápita. Además, el peso en las economías del sector servicios va de $54.73 \%$ a niveles de $92.83 \%$, indicando que existe actualmente una mayor relevancia de los servicios en la actividad económica a nivel global. En el periodo considerado, se ha registrado una alta demanda de combustibles de origen fósil en la generación de energía, con elevados porcentajes de participación en el consumo de energía entre 68 y 100 por ciento, por otra parte se registra en promedio una baja eficiencia en el uso de la energía, así como un bajo porcentaje de territorio declarados como áreas protegidas, lo cual es un indicador de la importancia que asignan los gobiernos en promover fuentes de energía alternativas y en la protección de los recursos naturales.

\footnotetext{
${ }^{8}$ La información estadística se obtuvo del sistema de Indicadores del Desarrollo Mundial del Banco Mundial, en http://databank.bancomundial.org

${ }^{9}$ En términos macroeconómicos, la forma habitual de medir la eficiencia energética es a través de la intensidad energética (IE). Este indicador refleja la relación entre consumo energético y el volumen de la actividad económica y se calcula como el cociente entre el consumo energético y el pIB. Por tanto, es el inverso de la eficiencia energética, siendo necesario disminuir la intensidad para mejorar la eficiencia energética.
} 


\section{Cuadro I}

Estadísticos de las variables 1990-2010

\begin{tabular}{|l|c|c|c|c|c|}
\hline \multicolumn{1}{|c|}{ Variable } & Media & Mediana & Max & Min & Dev. Std. \\
\hline $\mathrm{CO}_{2 \text { it }}$ & 5.10 & 3.42 & 38.16 & 0.02 & 5.50 \\
\hline$y p_{i t}$ & 10,525 & 3,210 & 87,717 & 112 & 14,781 \\
\hline serv $_{i t}$ & 54.73 & 55.01 & 92.83 & 15.90 & 13.54 \\
\hline $\mathrm{ffe}_{i t}$ & 67.59 & 75.98 & 100.00 & 2.70 & 28.20 \\
\hline efe $_{i t}$ & 7.69 & 7.57 & 24.43 & 0.98 & 3.76 \\
\hline aprot $_{i t}$ & 11.94 & 9.68 & 54.86 & 0.00 & 9.91 \\
\hline
\end{tabular}

Fuente: Elaboración propia con base en información de Banco Mundial

Gráfica I

Diagrama de dispersión emisiones

$\mathrm{CO}_{2}$ vs PIB per cápita: $1990-2010$

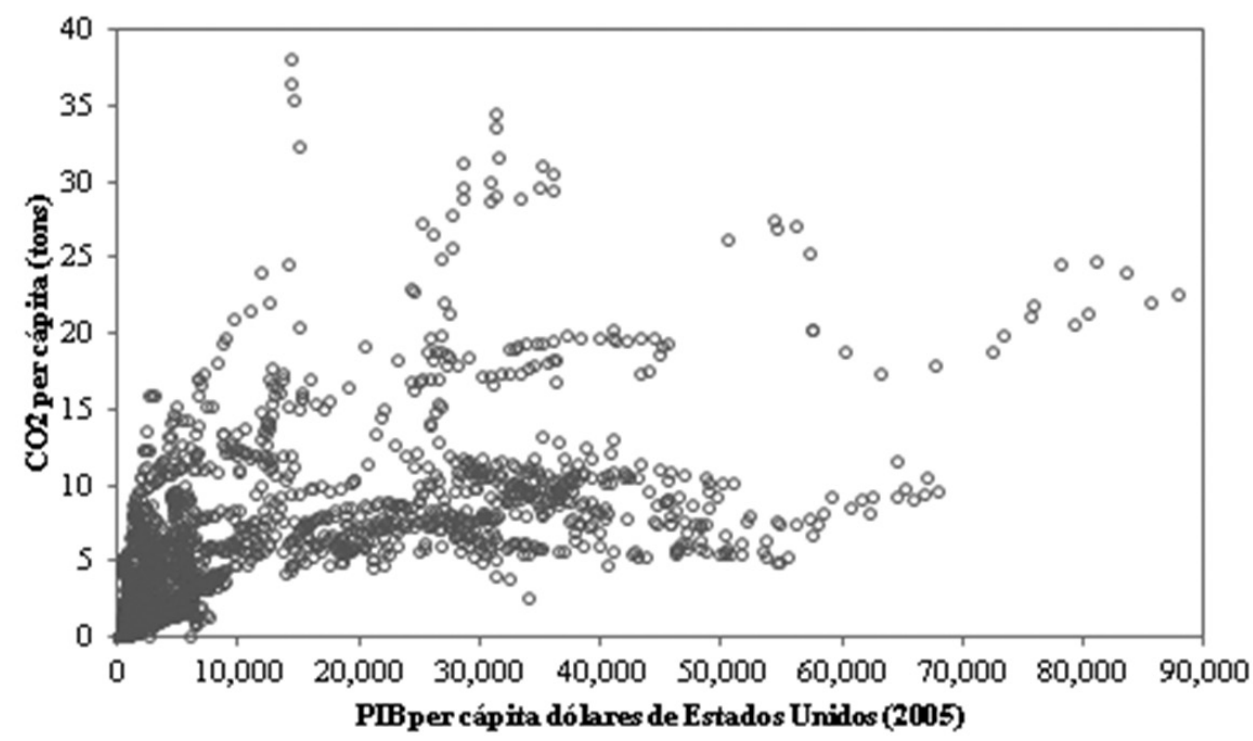

Fuente: elaboración propia con base en información de Banco Mundial.

La Gráfica 1 presenta un diagrama de dispersión entre las emisiones per cápita de $\mathrm{CO}_{2}$ y el PIB per cápita, se aprecia que un aumento del PIB per cápita está asociado a un amento en el nivel de emisiones por persona. Sin embargo, el patrón de correlación entre las dos variables deja de ser lineal al pasar de un valor de PIB per cápita superior a los 10 mil dólares. En efecto en valores 
superiores a este nivel, las emisiones de $\mathrm{CO}_{2}$ en algunos casos permanecen constantes entre 5 y 10 toneladas por persona, para otros países las emisiones se elevan a valores superiores a 10 toneladas por habitante. Niveles de PIB per cápita superiores a 20 mil dólares al año, no corresponden con una disminución de las emisiones de $\mathrm{CO}_{2}$, lo cual sería evidencia empírica en contra de una forma de U-invertida de la EKC, y en favor de un patrón no lineal en forma de N (Figura 2). Es decir, las economías en desarrollo mantienen una fuerte asociación entre crecimiento económico y emisiones, mostrando una trayectoria creciente. Por su parte, los países más desarrollados con mayores niveles de ingreso no muestran una reducción en las emisiones per cápita, de hecho estas tienden a mantenerse constantes.

Si bien la Gráfica 1, aporta elementos de discusión de la hipótesis de la EKC, no permite medir los impactos del crecimiento económico (aproximado por el PIB per cápita) y otras variables de control, por lo cual la comprobación empírica se realiza por medio de estimar una ecuación con datos panel. En primera instancia se realizó una estimación considerando únicamente las emisiones de $\mathrm{CO}_{2}$ y el PIB per cápita en nivel, al cuadrado y al cubo, es decir una versión simple de la hipótesis EKC, los resultados se presentan en el Cuadro 2. La estimación se realizó por mínimos cuadrados ordinarios (POLS), efectos fijos (FE) utilizando transformación within, efectos aleatorios por FGLS (RE), estimación Between (BE) y en primeras diferencias (FD). En las cinco especificaciones, los signos de los coeficientes del PIB per cápita cumplen con la restricción 4) es decir: $\beta_{1}>0, \beta_{2}<0$ y $\beta_{3}>0$, indicando que la relación emisiones de $\mathrm{CO}_{2}$ y PIB per cápita se puede aproximar por una muestra una curva en forma de N. Esto implica que, en un rango de bajos niveles de ingreso per cápita la relación creciente cambia a decreciente en un punto de inflexión, pero después de cierto nivel de ingreso, la relación entre ambas variables es positiva y posteriormente se mantiene constante.

La curva en forma de $\mathrm{N}$ indicaría que los beneficios que se puedan lograr, en cuanto a la disminución de emisiones de $\mathrm{CO}_{2}$, debido a un mayor crecimiento económico y un aumento en la eficiencia tecnológica son transitorias. Las opciones de reducción de emisiones se agotan o bien sus costos tienden a incrementarse, y en consecuencia no se logran reducciones significativas en la degradación ambiental. Por otra parte, el grado de ajuste de los modelos medidos por el estadístico R-cuadrado es bastante bajo, además del ingreso per cápita existen otros factores que determinan la evolución de las emisiones por habitante. En este sentido, se especió una ecuación de la EKC en una versión 


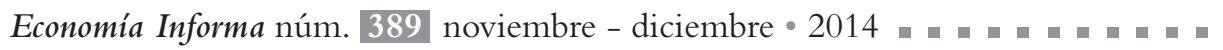

extendida que considera algunas variables de control ${ }^{10}$ como: la participación porcentual del sector servicios en el PIB total $\left(\operatorname{serv}_{i t}\right)$; el porcentaje de consumo de energía de origen fósil respecto al total de consumo de energía total ( $\mathrm{ffe}_{i t}$ ), la variable de eficiencia energética $\left(\mathrm{efe}_{i t}\right)$, la cual se define como el reciproco de la intensidad energética; ${ }^{11}$ y el porcentaje de áreas protegidas respecto al total del territorio $\left(\right.$ aprot $\left._{i t}\right)$.

\section{Cuadro 2}

\section{Estimación de la hipótesis simple de EKC}

\begin{tabular}{|c|c|c|c|c|c|}
\hline Variable & POLS & $\mathrm{FE}$ & $\mathrm{RE}$ & $\mathrm{BE}$ & FD \\
\hline$y p_{i t}$ & $\begin{array}{c}0.0009414 \\
(0.0000301) *\end{array}$ & $\begin{array}{c}0.0006915 \\
(0.000296) *\end{array}$ & $\begin{array}{c}0.0007283 \\
(0.0002172) *\end{array}$ & $\begin{array}{c}0.0010988 \\
(0.0001529) *\end{array}$ & $\begin{array}{c}0.0006687 \\
(0.0000912) *\end{array}$ \\
\hline$y p_{i t}{ }^{2}$ & $\begin{array}{c}-2.63 \mathrm{E}-08 \\
(1.20 \mathrm{E}-09) *\end{array}$ & $\begin{array}{c}-1.62 \mathrm{E}-08 \\
(6.20 \mathrm{E}-09) *\end{array}$ & $\begin{array}{c}-1.69 \mathrm{E}-08 \\
(4.92 \mathrm{E}-09) *\end{array}$ & $\begin{array}{l}-3.40 \mathrm{E}-08 \\
(6.44 \mathrm{E}-09) *\end{array}$ & $\begin{array}{l}-1.24 \mathrm{E}-08 \\
(2.14 \mathrm{E}-09) *\end{array}$ \\
\hline$y p_{i t}{ }^{3}$ & $\begin{array}{c}2.22 \mathrm{E}-13 \\
(1.17 \mathrm{E}-14) *\end{array}$ & $\begin{array}{c}1.08 \mathrm{E}-13 \\
(4.01 \mathrm{E}-14) *\end{array}$ & $\begin{array}{c}1.13 \mathrm{E}-13 \\
(3.37 \mathrm{E}-14) *\end{array}$ & $\begin{array}{c}3.10 \mathrm{E}-13 \\
(6.60 \mathrm{E}-14) *\end{array}$ & $\begin{array}{c}7.6 \mathrm{E}-14 \\
(1.52 \mathrm{E}-14) *\end{array}$ \\
\hline C & $\begin{array}{c}0.7275622 \\
(0.1221624) *\end{array}$ & $\begin{array}{c}1.631534 \\
(1.690946)\end{array}$ & $\begin{array}{c}1.459549 \\
(1.113001)\end{array}$ & $\begin{array}{c}0.4195696 \\
(0.5636374)\end{array}$ & $\begin{array}{c}-0.030087 \\
(0.0158661)\end{array}$ \\
\hline$R^{2}$ & 0.5249 & 0.4952 & 0.4967 & 0.5134 & 0.0260 \\
\hline $\mathrm{F}$ & $877.1667 *$ & $393.2557 *$ & $128.9470 *$ & $47.39 *$ & $21.1765 *$ \\
\hline
\end{tabular}

Nota: entre paréntesis el error estándar del estimador. (*) Significancia a un nivel de 5\%. En FD (primera diferencia) las variables se incluyen en primera diferencia.

El Cuadro 3, reporta los resultados de la estimación de la EKC-extendida en las cinco especificaciones para el modelo de datos panel. Los coeficientes del PIB per cápita son estadísticamente significativos y confirman la curva en forma de $\mathrm{N}$ al igual que en la versión simple. Las variables de control consideradas, con excepción del porcentaje de áreas protegidas ( aprot $\left._{i t}\right)$, también resultaron estadísticamente significativas en las cinco especificaciones. Las áreas protegidas son estadísticamente significativas solo en las especificaciones de efectos fijos y efectos aleatorios, que consideran la heterogeneidad no observada en las

\footnotetext{
${ }^{10}$ Inicialmente se consideraron otras variables como la densidad de población; la tasa de crecimiento del PIB y la intensidad del comercio internacional. Estas variables no resultaron estadísticamente significativas.

${ }^{11}$ En términos macroeconómicos, la forma habitual de medir la eficiencia energética es a través de la intensidad energética (IE). Este indicador refleja la relación entre consumo energético y el volumen de la actividad económica y se calcula como el cociente entre el consumo energético y el PIB. Por tanto, es el inverso de la eficiencia energética, siendo necesario disminuir la intensidad para mejorar la eficiencia energética.
} 
observaciones de sección cruzada. Al incluir las variables de control el grado de ajuste mejora de acuerdo a los valores del R-cuadrado, así que una versión ampliada de la EKC tiene una mejor capacidad de explicación y permite validar con mayor robustez la curva en forma de $\mathrm{N}$.

La participación porcentual del sector servicios en el PIB $\left(\operatorname{sev}_{i t}\right)$ registra un signo negativo en todas las especificaciones, esto es compatible con el efecto en la composición del producto (Grossman y Kruger, 1995), en favor de sectores como el de servicios que utilizan tecnologías más limpias, un usos más eficiente de la energía o fuentes alteñas de energía (Stern, 2003), que se traducen en menores emisiones de $\mathrm{CO}_{2}$. En contraste el consumo de energía de origen fósil (ffe ${ }_{i t}$ )reporta un signo positivo, lo cual es consistente considerando que un mayor consumo de combustibles de origen fósil como el petróleo genera un mayor deterioro ambiental. Es importante señalar que la quema de combustibles de origen fósil es la principal fuente de emisiones de $\mathrm{CO}_{2}$, asociados a la demanda de energía de la industria y el transporte, principalmente. Además factores como el precio del petróleo y las políticas energéticas en favor de combustibles de origen fósil inducen un mayor consumo y en consecuencia mayores emisiones.

La variable de eficiencia energética resulto negativa y estadísticamente significativa, pero además la magnitud del coeficiente muestra una relevancia importante en la trayectoria de las emisiones de $\mathrm{CO}_{2}$. En efecto, la eficiencia energética es una de las principales variables que inducen una reducción en el consumo de energía de origen fósil y permiten desacoplar la trayectoria de la actividad económica y el producto. En un estudio realizado por la Agencia Internacional de Energía (IEA, 2008) muestra que sin las mejoras realizadas en materia de eficiencia energética durante el periodo de 1973 a 2005 a nivel global, el uso de energía habría sido 58\% mayor al nivel registrado en 2005, es decir la eficiencia energética genera reducciones importantes en la demanda de energía. Sin embargo, desde 1990, la tasa de eficiencia energética se estancó debido al menor interés económico debido al precio relativamente bajo de los combustibles induciendo un aumento en la demanda del petróleo. 
Economía Informa núm. 389 noviembre - diciembre • 2014 - "

\section{Cuadro 3}

\section{Estimación de la EKC con variables de control}

\begin{tabular}{|c|c|c|c|c|c|}
\hline Variable & POLS & $\mathrm{FE}$ & $\mathrm{RE}$ & $\mathrm{BE}$ & FD \\
\hline$y p_{i t}$ & $\begin{array}{c}0.0008427 \\
(0.000028) *\end{array}$ & $\begin{array}{c}0.0010564 \\
(0.0003226) *\end{array}$ & $\begin{array}{c}0.0009318 \\
(0.0002112) *\end{array}$ & $\begin{array}{c}0.001025 \\
(0.0001443) *\end{array}$ & $\begin{array}{c}0.0007862 \\
(0.0000927) *\end{array}$ \\
\hline$y p_{i t}{ }^{2}$ & $\begin{array}{c}-2.14 \mathrm{E}-08 \\
(1.04 \mathrm{E}-09) *\end{array}$ & $\begin{array}{c}-2.19 \mathrm{E}-08 \\
(6.34 \mathrm{E}-09) *\end{array}$ & $\begin{array}{c}-1.96 \mathrm{E}-08 \\
(4.73 \mathrm{E}-09) *\end{array}$ & $\begin{array}{c}-2.93 \mathrm{E}-08 \\
(5.65 \mathrm{E}-09) *\end{array}$ & $\begin{array}{c}-1.38 \mathrm{E}-08 \\
(2.12 \mathrm{E}-09) *\end{array}$ \\
\hline$y p_{i t}^{3}$ & $\begin{array}{c}1.80 \mathrm{E}-13 \\
(9.84 \mathrm{E}-15) *\end{array}$ & $\begin{array}{c}1.41 \mathrm{E}-13 \\
(4.07 \mathrm{E}-14) *\end{array}$ & $\begin{array}{c}1.28 \mathrm{E}-13 \\
(3.3 \mathrm{E}-14) *\end{array}$ & $\begin{array}{c}2.66 \mathrm{E}-13 \\
(5.57 \mathrm{E}-14) *\end{array}$ & $\begin{array}{c}8.26 \mathrm{E}-14 \\
(1.49 \mathrm{E}-14) *\end{array}$ \\
\hline $\operatorname{serv}_{i t}$ & $\begin{array}{c}-0.0736493 \\
(0.0056931) *\end{array}$ & $\begin{array}{c}-0.0396427 \\
(0.0145098) *\end{array}$ & $\begin{array}{c}-0.0410917 \\
(0.0158197) *\end{array}$ & $\begin{array}{c}-0.1007584 \\
(0.0283437) *\end{array}$ & $\begin{array}{c}-0.0123545 \\
(0.0046323) *\end{array}$ \\
\hline $\mathrm{ffe}_{i t}$ & $\begin{array}{c}0.0728413 \\
(0.0024764) *\end{array}$ & $\begin{array}{c}0.0352531 \\
(0.0097577) *\end{array}$ & $\begin{array}{c}0.0402616 \\
(0.0091543) *\end{array}$ & $\begin{array}{c}0.0713002 \\
(0.0112415) *\end{array}$ & $\begin{array}{c}0.0319366 \\
(0.0070545) *\end{array}$ \\
\hline $\mathrm{efe}_{i t}$ & $\begin{array}{c}-0.4380808 \\
(0.0169379) *\end{array}$ & $\begin{array}{c}-0.373903 \\
(0.0917459) *\end{array}$ & $\begin{array}{c}-0.3491107 \\
(0.0726192) *\end{array}$ & $\begin{array}{c}-0.4500987 \\
(0.0772166) *\end{array}$ & $\begin{array}{c}-0.2986656 \\
(0.0281151) *\end{array}$ \\
\hline $\operatorname{aprot}_{i t}$ & $\begin{array}{c}0.0110478 \\
(0.0061133)\end{array}$ & $\begin{array}{c}-0.0337659 \\
(0.0153091) *\end{array}$ & $\begin{array}{c}-0.0266591 \\
(0.0117665) *\end{array}$ & $\begin{array}{c}0.0102351 \\
(0.0277433)\end{array}$ & $\begin{array}{l}-0.0013471 \\
(0.0060387)\end{array}$ \\
\hline $\mathrm{C}$ & $\begin{array}{c}3.116834 \\
(0.3058392) *\end{array}$ & $\begin{array}{c}2.278792 \\
(0.9399909) *\end{array}$ & $\begin{array}{c}2.524679 \\
(0.7105072) *\end{array}$ & $\begin{array}{c}4.317801 \\
(1.470378) *\end{array}$ & $\begin{array}{l}-0.0139747 \\
(0.0158218)\end{array}$ \\
\hline$R^{2}$ & 0.7341 & 0.6467 & 0.6668 & 0.7227 & 0.0992 \\
\hline F & $926.94 *$ & $472.65 *$ & $167.64 *$ & $51.75 *$ & $35.18 *$ \\
\hline
\end{tabular}

Nota: entre paréntesis el error estándar del estimador. (*) Significancia a un nivel de 5\%. En FD (primera diferencia) las variables se incluyen en primera diferencia.

Finalmente el porcentaje de áreas protegidas ( $\left.\operatorname{aprot}_{i t}\right)$, sólo fueron significativas en las especificaciones de efectos fijos y efectos aleatorios con signo negativo. Esta variable puede ser considerada como una variable proxy de la política ambiental a nivel país o una variable que mide las preferencias de las sociedades a la protección ambiental, toda vez que al medir el área (terrestre y marítima) destinada a la protección y conservación, es una aproximación al grado de respeto a los recursos naturales y la biodiversidad. Un mayor porcentaje de área protegida esta correlacionada con un mayor grado de compromiso a la protección del medio ambiente y en consecuencia con un menor nivel de emisiones. El signo negativo refleja esta relación, es decir un mayor porcentaje de área protegida muestran una mayor información y educación sobre las consecuencias ambientales, y un mayor compromiso de los países en la disminución de las emisiones

Las especificaciones de efectos fijos y efectos aleatorios en la EKC incluyendo las variables de control, resultaron consistentes con la hipótesis teórica, 
y en términos de los signos y magnitudes de los estimadores. La prueba estadística de Hausman (Baltagi, 2005) puede ser utilizada para determinar la mejor especificación entre efectos fijos y aleatorios. Esta prueba compara los estimadores de efectos fijos y efectos aleatorios, y si existe una diferencia que sea estadísticamente significativa entre ambos, indica que los estimadores de efectos aleatorios son inconsistentes en consecuencia la mejor especificación para el modelo de datos panel corresponde a la de efectos fijos (Hsiao, 2003, Baltagi, 2005, Greene, 2000).

Se asume como hipótesis nula que las variables explicativas no están correlacionadas con la heterogeneidad no observada $H_{0}: E\left(x_{i t}{ }^{\prime} \alpha_{i}\right)=0$ y la alternativa $H_{0}: E\left(x_{i t}{ }^{\prime} \alpha_{i}\right) \neq 0$. Rechazar la hipótesis nula es un argumento a favor de la especificación de efectos fijos (Hsiao, 2003). El estadístico de la prueba se define con base en la diferencia de los estimadores de efectos fijos y aleatorios $\hat{q}=\hat{\beta_{F E}}-\hat{\beta_{R E}}$, y la diferencia en el valor de las varianzas de los estimadores $\operatorname{Var}(\hat{q})=\operatorname{Var}\left(\hat{\beta}_{F E}\right)-\operatorname{Var}\left(\hat{\beta}_{R E}\right)$. Finalmente el estadístico de Hausman se define como: $m=\hat{q}^{\prime}[\operatorname{Var}(\hat{q})]^{-1} \hat{q}$, el cual se distribuye como una ji-cuadrada con $\mathrm{k}$ grados de libertad $\chi^{2}(k)$, que es el número de parámetros. El Cuadro 4, presenta los resultados de la prueba de Hausman para la estimación de la EKC simple y la EKC con variables de control, en ambos casos se rechaza la hipótesis nula, indicando correlación entre las variables del modelo y los efectos individuales, por lo tanto la mejor especificación en datos panel es la de efectos fijos.

\section{Cuadro 4}

\section{Resultados de la Prueba de Hausman}

\begin{tabular}{|c|c|}
\hline Modelo & Estadístico \\
\hline EKC-Simple & $\chi^{2}(3)=15.9863(0.001)$ \\
\hline EKC-extendida & $\chi^{2}(7)=61.6002(0.000)$ \\
\hline
\end{tabular}

Nota: entre paréntesis probabilidad de rechazo de la hipótesis nula.

\section{Conclusiones}

La evidencia empírica presentada en este trabajo muestra que al considerar una muestra de 144 países en el periodo de 1990 a 2010, mediante un modelo de datos panel, la relación entre las emisiones per cápita de $\mathrm{CO}_{2}$ y el PIB per cápita se ajusta a una curva ambiental de Kuznets en forma de N, en la cual los países con bajo ingreso per cápita tienden a elevar las emisiones per cápita y con ello el deterioro ambiental, debido a que la explotación de los recursos 
Economía Informa núm. 389 noviembre - diciembre • 2014 - "

naturales se realiza con las tecnologías no eficientes y la extracción de los recursos naturales excede a su conservación. Por otra parte, las variables de control muestran que en estos países la estructura de la economía se concentra en la agricultura y en la industria, con alto consumo de combustibles de origen fósil, bajos niveles de eficiencia energética, así como una escasa protección de los recursos naturales y la biodiversidad.

Los países con un alto ingreso per cápita, muestran una reducción de los niveles de emisiones con una estructura de la economía apoyada principalmente en los servicios, una mejor eficiencia energética, moderados niveles de consumo de combustibles de origen fósil y una mayor preocupación por los temas ambientales. Sin embargo, la estimación muestra que la curva EKC tiene forma de $\mathrm{N}$, por lo tanto en países con altos niveles de ingreso las reducción de las emisiones se detiene, posiblemente a que las oportunidades de reducir emisiones son cada vez menores, los costos de reducción tiende a levarse debido a que el precio de las nuevas tecnologías son más elevados. También, se genera un traslado de los de los procesos de producción que generan emisiones, de países de mayor ingreso hacia países que no aplican una regulación estricta en materia ambiental (Rothman, 1998).

Estos resultados muestran que existen implicaciones importantes para lograr un crecimiento sustentable en el tiempo. En primer lugar, no puede argumentarse que es posible "salir" de los problemas ambientales exclusivamente a través del crecimiento económico y en consecuencia las políticas regulatorias en materia ambiental y energética juegan un papel relevante en revertir el deterioro ambiental. Las estimaciones de la EKC incluyendo variables de control, muestran que las mejoras ambientales no dependen solo del crecimiento económico, la eficiencia energética es una variable relevante en el diseño de políticas para lograr reducir las emisiones, así como la protección a la biodiversidad y conservación de áreas naturales. Por tanto se requiere la instrumentación de regulaciones adecuadas y de mantener un continuo proceso de innovación tecnológica.

En efecto, la presencia de un coeficiente negativo y estadísticamente significativo en la variable de eficiencia energética, indica que el progreso técnico, en el contexto de una regulación adecuada, es fundamental para reducir los niveles de contaminación (Anderson y Cavendish, 2001) ya que incluye cambio en la combinación de combustibles, fuentes de energía alternativas, y un creciente gasto en investigación y tecnología como proporción del PIB (Komen, Gerking y Folmer, 1997). Debe sin embargo, considerarse a este respecto que existen importantes rezagos entre el inicio de una política y sus 
impactos. Por ejemplo, se estima que la maquinaria y equipo dura en general aproximadamente 25 años lo que, desde luego, retrasa la obtención de impactos favorables al medio ambiente (Anderson y Cavendish, 2001).

De este modo, el problema ambiental no se reduce a la evolución del crecimiento económico (Arrow, et al, 1995) sino que debe considerarse el papel de los mercados, la innovación tecnológica y las regulaciones ambientales. Finalmente, debe considerarse que las regulaciones ambientales tienden a ser más estrictas en un contexto donde aquellos que son afectados por la degradación ambiental tienen mecanismos claros de defensa y compensación (Torras y Boyce, 1998). Por tanto, no es exclusivamente en los períodos de crecimiento económico en donde las regulaciones ambientales son efectivas sino que debe considerarse la capacidad institucional de los gobiernos para lograr reducciones significativas en el deterioro ambiental.

\section{Bibliografía}

Andreoni J. y A. Levinson (2001), "The simple analytics of the environmental Kuznets curve", Journal of Public Economics, 80(2), pp. 269-286

Arrow, K., B. Bolin, R. Costanza, P. Dasgupta, C. Folke, C.S. Holling, B.-O.

Jansson, S. Levin, K.-G. Maler, C.Perrings y D. Pimentel (1995), "Economic growth, carrying capacity and the environment", Ecological Economics, 15(2), pp. 91-95.

Baltagi B. H. (2005), Econometrics, $3^{\text {a }}$ edición, Alemania, Springer-Verlag

Beckerman W. (1992), "Economic Growth and the Environment: Whose Growth? Whose Environment?”, World Development, 20(4), pp. 481-496

Bruyn de, S.M. (1997), "Explaining the environmental Kuznets curve: structural change and international agreements in reducing sulphur emissions", Environment and Development Economics, 2, pp. 485-503.

Bruyn de, S.M., J.C.J.M. Bergh y J.B. Opschoor (1998), "Economic growth and emissions: reconsidering the empirical basis of environmental Kuznets curve", Ecological Economics, 25, pp. 161-175.

Choumert J., Combes-Motel P., y Dakpo H. K., (2013), Is the Environmental Kuznets Curve for deforestation a threatened theory? A meta-analysis of the literature, Ecological Economics, 90, pp. 19-28

Dinda S., (2004), "Environmental Kuznets Curve Hypothesis: A Survey”, Ecological Economics, 49(4), pp. 431-455

Dinda S. (2005), "A theoretical basis for the environmental Kuznets curve", Ecological Economics, 53, pp. 403-413 


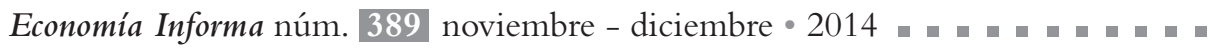

Ekins, P. (1997), "Kuznets curve for the environment and economic growth: examining the evidence", Environmental and Planning Annals, 29, pp. 805-830.

Galeotti, M., L. Lanza y F. Pauli, (2006), "Reassessing the environmental Kuznets curve for CO2 emissions: A robustness exercise", Ecological Economics, 57, pp. $152-163$

Goldemberg, J. (1992), "Energy, technology, development”, Ambio, 21, 14-17.

Greene, W.H. (2000), Econometric Analysis, 4ª edición, Upper Saddle River, NJ: Prentice Hall

Grossman, Gene M., y Alan B. Krueger. (1995), "Economic Growth and the Environment”, The Quarterly Journal of Economics, 110(2), pp. 353-377

Grossman, G.M. y A.B. Krueger (1993), "Environmental impacts of a North American Free Trade Agreement", in The U.S.-Mexico Free Trade Agreement, P. Garbe, ed.

Grossman, Gene M., y Alan B. Krueger (1991). "Environmental Impact of a North American Free Trade Agreement". Working Paper 3914. National Bureau of Economic Research, Cambridge, MA

He J. y H. Wang (2012), "Economic structure, development policy and environmental quality: An empirical analysis of environmental Kuznets curves with Chinese municipal data", Ecological Economics, 76, pp. 49-59

Hsiao C. (2003), Analysis of Panel Data, $2^{\mathrm{a}}$ edición, Estados Unidos, Cambridge University Press

IEA (2008), Worldwide Trends in Energy Use and Efficiency. Key Insights from IEA Indicator Analysis, IEA, Paris, IEA (2011a), Energy Statistics of OECD Countries and Non-OECD Countries, 2011 Edition. International Energy Agency, Paris.

Kaufmann R. K., B. Davidsdottir, S. Garnham y P. Pauly (1998), “The determinants of atmospheric SO2 concentrations: reconsidering the environmental Kuznets curve", Ecological Economics, 25, pp. 209-220.

Komen, M.H., S. Gerking y H. Folmer (1997), "Income and environmental R\&D: empirical evidence from OECD countries", Environment and Development Economics, 2, pp. 505-515.

Kuznets, S. (1955), "Economic growth and income inequality", American Economic Review, 49, 1-28.

Lessmann C. (2014), "Spatial inequality and development-Is there an inverted-U relationship?”, Journal of Development Economics, 106, pp. 35-51

McConnell (1997), "Income and the demand for environmental quality", Environment and Development Economics, 2, pp. 383-399. 
Moomaw, W.R. y G. Unruh (1997), "Are environmental Kuznets curves misleading us? The case of CO2 emissions", Environment and Development Economics, 2, pp. 451-463.

Nahman A. y G. Antrobus (2005), “The environmental Kuznets curve: a literature survey", South African Journal of Economics, 73(1), pp. 105-120.

Panayotou, T. (1997), "Demystifying the environmental Kuznets curve: turning a black box into a policy tool", Environment and Development Economics, 2, pp. 465-484.

Panayotou, T. (1993). "Empirical Tests and Policy Analysis of Environmental Degradation at Different Stages of Economic Development". Working Paper WP238, Technology and Employment Programme, International Labour Office, Geneva.

Perman, R. J., Y. Ma, J. McGilvray y M. Common (2003), Natural Resource and Environmental Economics (Third edition), Addison Wesley Longman.

Rothman, D.S. y S. M. de Bruyn (1998), "Probing into the environmental Kuznets curve hypothesis", Ecological Economics, 25, pp. 143-145.

Rothman, D. S. (1998), "Environmental Kuznets curves: real progress or passing the buck?: a case for consumption-based approaches", Ecological Economics, 25, pp. 177-194.

Selden, T.M., Song, D. (1994). "Environmental quality and development: is there a Kuznets curve for air pollution emissions?", Journal of Environmental Economics and Management, 27, pp. 147-162.

Stern, D.I. (2003), “The Environmental Kuznets Curve”, International Society for Ecological Economics, Working Papers.

Stern, D.I. y Common, M.S. (2001), "Is there an environmental Kuznets curve for sulphur?", Journal of Environmental Economics and Management, 41, pp. 162-178.

Stern, D. I. (1998). "Progress on the environmental Kuznets curve?", Environment and Development Economics, 3(2), pp. 173-196.

Stern, D. y M. Common (1996), "Economic growth and environmental degradation: the enviromental Kuznets curve and sustainable development", World Development, 24(7), 1151-1160.

Suri, V. y D. Chapman (1998), "Economic growth, trade, and energy: implications for the environmental Kuznets curve", Ecological Economics, 25, 195-208.

Tilton, J.E. (1990), "World metal demand: trends and prospects", en Resources for the Future, Washington, D. C, pp. 25-30

Torras, M. y J.K. Boyce (1998), "Income, inequality, and pollution: a reassessment of the environmental Kuznets curve", Ecological Economics, 25, 147-160. 